\title{
El modelo secuencial en el análisis de políticas públicas: el caso de la política pública en investigación científica y tecnológica de la UNAH
}

Jorge Edgardo Amador ${ }^{1}$

\section{INTRODUCCIÓN}

El 12 de febrero del 2005 se publica en el Diario oficial La Gaceta la nueva ley orgánica de la Universidad Nacional Autónoma de Honduras, la cual fue concebida como un punto de partido para alcanzar una reforma integral a una Universidad Nacional que necesitaba potenciarse para mantener su liderazgo en el contexto nacional e internacional tal como lo manda el artículo 160 de la Constitución Política de Honduras.

Tal como lo señala Olvin Rodríguez, ex presidente de la Junta de Dirección Universitaria en una columna del Diario El Heraldo (s.f.) denominada "Algunas causas de la crisis en la UNAH", expone que una de ellas priorizándola ante las demás es la legitimidad del gobierno universitario:

\footnotetext{
"Hay que ponderar que antes del 2004 la UNAH ya tocaba fondo. Una Ley Orgánica que concentraba el poder en la Rectoría y unas autoridades, frentes estudiantiles y dirigencia del SITRAUNAH, en una especie de cofradía, usufructuaban la institución y la llevaron al desastre; el gobierno universitario coludido, cedió la responsabilidad de dirección, gestión y conducción al sindicato, al grado que no se sabía dónde empezaba la responsabilidad del gobierno universitario".
}

Esta causa, y debido a la magnitud y responsabilidad constitucional que representa la Universidad Nacional Autónoma de Honduras, el Congreso Nacional de la Republica, determino que se requería un nuevo instrumento normativo que contrarrestara las debilidades de los instrumentos anteriores: más que una simple ley se comienza la construcción de nuevas instituciones en la UNAH. El artículo 61 de la Ley Orgánica de la UNAH promulga "Crease la Comisión de Transición para asegurar la reforma institucional integral de la UNAH, la organización de la nueva estructura universitaria, la instalación del nuevo gobierno universitario y, en general, la pronta y correcta aplicación de esta Ley". Como parte de este mandado que arguye la Ley, parte de la nueva estructura universitaria y del nuevo gobierno universitario es el sistema de investigación científica y tecnológica de la UNAH.

1 Profesor de la Dirección de Investigación Científica y Posgrado, UNAH: edgardo_honduras@hotmail.com 
Por instituciones entenderemos lo expuesto por Medrano (2000) como "la acción y el efecto de instituir o institucionalizar, esto es, fundar y establecer con firmeza" (pág. 67), una de estas instituciones es la reconstrucción de la función básica universitaria: la investigación científica. La Comisión de Transición institucionaliza y reivindicada la función básica de la investigación científica en el Plan General para la Reforma Integral de la Universidad emitido en el año 2005, identificando y describiendo las áreas e indicadores de crisis, en este caso solo se enfatizan las que corresponden a la función de investigación.Como quinta área de crisis descrita en el Plan General de la Reforma Integral de la Universidad, se encuentra la "Producción de pensamiento, de liderazgo social y de identidad nacional", desagregado en indicadores, los que corresponden a este tema son: a) la producción de conocimiento original, patentable, de carácter universal o bien orientado a la solución de problemas nacionales no ha sido atendida con prioridad y, b) la presencia de la Universidad como voz intelectual y científica de la nación se ha venido reduciendo paulatinamente a lo largo de los últimos años. (Comisión de Transición UNAH , 2005)

Esta área en crisis e indicadores requerían de la construcción y propuesta de un objetivo general que condujera el quehacer para mejorar tal crisis, por ello de los cinco objetivos generales que guiaron el Plan General de la Reforma Integral de la Universidad, uno corresponde a la función de la investigación científica, expresando y proponiendo "Contar en la UNAH con un sistema de investigación científica que contribuya al abordaje y superación de los grandes problemas nacionales, a democratizar el saber y a insertar positivamente a Honduras en la red mundial de producción, divulgación y gestión del conocimiento y de la información". (Comisión de Transición UNAH , 2005)

De los objetivos generales se desarrollan áreas pragmáticas y componentes de la reforma, atribuyendo la segunda área pragmática a la investigación y conocimiento. Esta área pragmática tuvo como objetivo general: "Desarrollar en la UNAH una capacidad científica endógena y de divulgación del conocimiento y de la técnica, en el marco de las posibilidades institucionales y del país, a fin de ayudar a Honduras a alcanzar un nivel mayor en Ciencia y Tecnología" (Comisión de Transición UNAH, 2005, pág. 62); este objetivo general del área pragmática fue acompañado de un objetivo estratégico, proponiendo: "Desarrollar interés, compromiso y capacidad de investigación en la comunidad universitaria, en áreas temáticas claves para el desarrollo hondureño; y lograr una inversión acorde con los requerimientos del desarrollo del sistema de investigación universitario" (Comisión de Transición UNAH , 2005, pág. 62). 


\section{Tabla 1. Indicadores de impacto y resultados esperados del área pragmática y componentes de Investigación y conocimiento del Plan General de la Reforma Integral de la Universidad 2005}

\begin{tabular}{|c|c|}
\hline Indicadores de impacto & Resultados esperados al 2007 \\
\hline $\begin{array}{l}\text { Implementación del Sistema de Investigación } \\
\text { Científica de la UNAH, con un conjunto de políticas, } \\
\text { estrategias, procesos y proyectos relacionados } \\
\text { entre sí y vinculados con las necesidades del } \\
\text { desarrollo nacional, local y regional e integrado al } \\
\text { sistema nacional de ciencia y tecnología. }\end{array}$ & \multirow{2}{*}{$\begin{array}{l}\text { Funcionando con calidad y pertinencia el } \\
\text { sistema de investigación de la UNAH, } \\
\text { apoyado en un sistema de incentivos } \\
\text { para la investigación y articulado con el } \\
\text { sistema nacional de ciencia y tecnología. } \\
\text { En ejecución proyecto de equipamiento } \\
\text { en apoyo al sistema de investigación de } \\
\text { la UNAH. }\end{array}$} \\
\hline $\begin{array}{l}\text { En funcionamiento un Sistemas de Incentivos para } \\
\text { la investigación }\end{array}$ & \\
\hline $\begin{array}{l}\text { La catedra de Estudios de la Realidad Nacional } \\
\text { creada y en funcionamiento, con apoyo de la } \\
\text { UNESCO }\end{array}$ & $\begin{array}{l}\text { Desarrollándose la catedra de la realidad } \\
\text { nacional en Tegucigalpa y en los Centros } \\
\text { Universitarios Regionales }\end{array}$ \\
\hline $\begin{array}{l}\text { Eje de educación en investigación integrado en los } \\
\text { planes de estudio de todas las carreras de la } \\
\text { UNAH. }\end{array}$ & $\begin{array}{l}\text { Implementándose el eje de educación en } \\
\text { investigación en todas las carreras de la } \\
\text { UNAH, como un componente de la } \\
\text { reforma curricular. }\end{array}$ \\
\hline $\begin{array}{l}\text { Vinculación permanente, planificada y sostenida de } \\
\text { la UNAH con el sector productivo para definir líneas } \\
\text { de investigación prioritarias. }\end{array}$ & \multirow{2}{*}{$\begin{array}{l}\text { Aprobada por la cooperación } \\
\text { internacional y con financiamiento del } \\
\text { sector productivo nacional una cartera de } \\
\text { proyectos de investigación en áreas } \\
\text { prioritarias para el desarrollo del país y el } \\
\text { combate a la pobreza. }\end{array}$} \\
\hline $\begin{array}{l}\text { En ejecución cartera de proyectos de investigación } \\
\text { con el apoyo de organizaciones de cooperación y } \\
\text { países amigos. }\end{array}$ & \\
\hline $\begin{array}{l}\text { Academia de Ciencias de la UNAH creada y en } \\
\text { funcionamiento. }\end{array}$ & $\begin{array}{l}\text { Diseñada de forma concertada la } \\
\text { propuesta de creación de la Academia de } \\
\text { Ciencias de la UNAH. }\end{array}$ \\
\hline \multicolumn{2}{|l|}{$\begin{array}{l}\text { Instituto de Investigación Educativa creado y en } \\
\text { funcionamiento }\end{array}$} \\
\hline $\begin{array}{l}\text { Instituto de Investigaciones en Ciencias de la tierra } \\
\text { en funcionamiento }\end{array}$ & $\begin{array}{l}\text { esta de creación del } \\
\text { laciones en Ciencias }\end{array}$ \\
\hline \multicolumn{2}{|l|}{$\begin{array}{l}\text { Programa de re vinculación de científicos } \\
\text { hondureños en el exterior diseñado y en } \\
\text { funcionamiento, con el apoyo de la UNESCO }\end{array}$} \\
\hline \multicolumn{2}{|l|}{$\begin{array}{l}\text { Programa de certificación de investigadores creado } \\
\text { y en funcionamiento }\end{array}$} \\
\hline \multicolumn{2}{|l|}{ Carrera de investigador universitario implementada } \\
\hline \multicolumn{2}{|l|}{$\begin{array}{l}\text { Participación de la UNAH en proyectos de } \\
\text { investigación colaborativos y multicéntricos }\end{array}$} \\
\hline $\begin{array}{l}\text { La editorial universitaria reorientada y apoyada } \\
\text { para que sustente la estrategia de divulgación del } \\
\text { conocimiento generado en la UNAH a nivel } \\
\text { nacional e internacional. }\end{array}$ & $\begin{array}{l}\text { En ejecución proyecto de fortalecimiento } \\
\text { de la Editorial Universitaria }\end{array}$ \\
\hline
\end{tabular}

Fuente: (Comisión de Transición UNAH , 2005) 


\section{El modelo secuencial para el análisis de políticas públicas}

El modelo secuencial junto al neo institucionalismo, la internacionalización de políticas públicas y al método cognitivo son un conjunto de enfoques que tienen una aplicabilidad desde la visión estatal, por ello se determinó la coyuntura de aplicar dicho modelo a la política de investigación científica y tecnológica de la UNAH. El modelo secuencial fue concebido por Lasswell (1956) y Almond \& Powell (1966) en The decision process: seven categories of funcional analysis y Comparative politics: a developmental approach respectivamente, teniendo como idea principal que la "política es un proceso que se desenvuelve por etapas, cada una posee sus actores, restricciones, decisiones, desarrollos, resultados propios, y se influyen mutuamente" (Pallavicini, 2016, pág. 3).

Por lo cual el modelo secuencial es conceptualizado por Peters (1992) citado en Estévez \& Esper (2009, pág. 77) como:

"un modelo genérico para la comprensión de políticas públicas, cuyo eje central es la idea de que la hechura de las políticas públicas (policy - making) se inicia con una fase de identificación del problema y, luego, atraviesa una serie de etapas que se espera concluyan en una evaluación, a partir de la cual podría tener lugar una reformulación y reinicio de la misma"

El modelo secuencial se sostiene en trece supuestos que pueden ser aplicados en su totalidad o de manera parcial según lo dispuesto en el proceso de análisis de la política pública, Jones (1970) citado en Estévez \& Esper (2009, pág. 78):

1. Los hechos en una sociedad son interpretados en forma variada por las diferentes personas y en múltiples momentos.

2. Muchos problemas pueden resultar a partir de un mismo hecho.

3. La gente goza de diferentes grados de acceso al proceso de las políticas en el gobierno.

4. No todos los problemas públicos son tenidos en cuenta por el gobierno.

5. Muchos problemas privados si son tenidos en cuenta por el gobierno.

6 . No todos los problemas son resueltos por el gobierno si bien muchos son tenidos en cuenta.

7. Los hacedores de políticas no necesariamente se enfrentan con un problema determinado.

8. Buena parte de la decisión está basada pequeñas transformaciones y en comunicaciones pobres.

9. Los problemas y las demandas son constantemente definidos y redefinidos en el 
proceso de las políticas públicas.

10. En ocasiones, los hacedores de políticas definen los problemas de gente que no los han definido por sí misma.

11. Muchas políticas públicas son hechas sin haber definido claramente el problema que ocasiona.

12. Todo sistema político tiene un sesgo.

13. No existe un sistema político ideal independiente de las preferencias del arquitecto de dicho sistema.

Estos trece supuestos se agrupan en etapas de política pública, tal como lo muestra la ilustración 1, y posteriormente se detalla cómo cada una de las etapas propuestas por Jones (1970) se intercepta con funciones o actividades funcionales que el gobierno practica en relación a las políticas públicas.

Es de tal forma que la intercepción y como se muestra en la tabla 2, se distribuyen en tres grandes grupos, las actividades para el gobierno, las actividades para el sistema y las actividades que posteriormente se constituyen en productos.

\section{Ilustración 1}

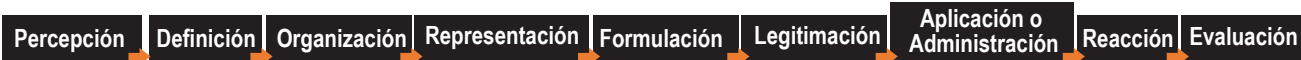

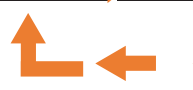

Fuente Estévez \& Esper (2009)

Aplicando el modelo secuencial en el Sistema de Investigación Científica y Tecnológica de la UNAH: la política de investigación científica, desarrollo tecnológico e innovación. UNAH 2015 - 2019

Antes de iniciar el análisis es necesario determinar ciertos aspectos. El primero determinar el órgano de gobierno. En este caso según lo descrito en la Ley Orgánica de la UNAH en su artículo 7, expone "el Consejo Universitario es el órgano máximo de dirección superior, responsable de concertar y generar las políticas y estrategias de la gestión universitaria, con visión de largo plazo, realizar contraloría social, evaluar y reorientar el cumplimiento de los objetivos transcendentales de la UNAH, velar por la transparencia, la autonomía universitaria y los intereses del Alma Mater..." 
Tabla 2: Modelo secuencial y los output (productos)

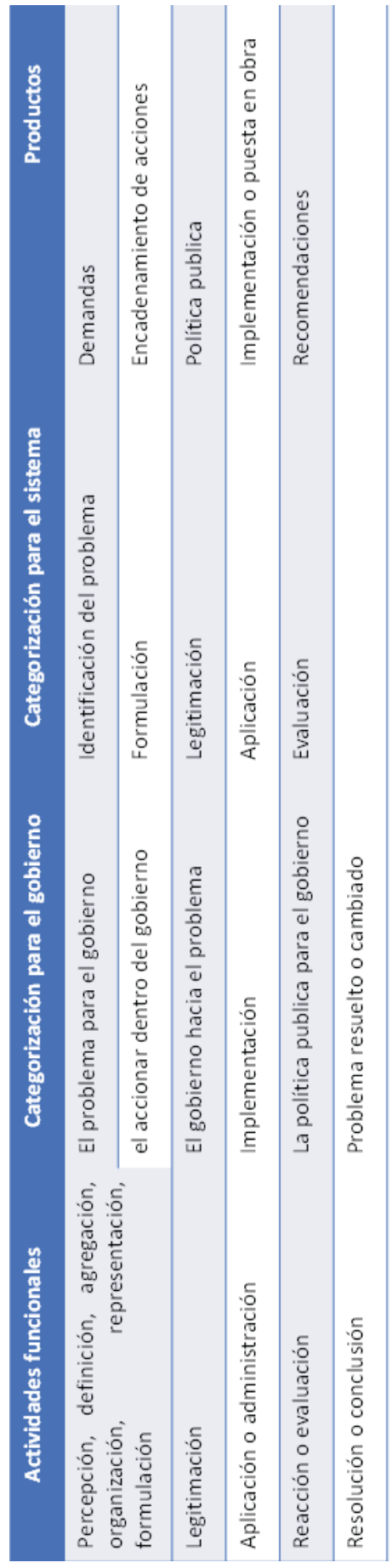

Fuente: Fuente Estévez \& Esper (2009) 
También es de aclarar que el sistema de investigación científica y tecnológica de la UNAH es creado mediante el Reglamento del Sistema de Investigación Científica y Tecnológica de la Universidad Nacional Autónoma de Honduras en el artículo 3, teniendo una estructura organizacional constituida por capas, y siendo su máximo órgano de conducción, coordinación, seguimiento y evaluación el Consejo General de Investigación Científica.

Después de revisar las funciones descritas en ambos documentos normativos, se define que el Consejo Universitario de la UNAH es el órgano de gobierno que legisla exclusivamente, a pesar de que el mismo (Consejo Universitario) ha delegado en el Consejo General de Investigación Científica ciertas funciones legislativas de primera instancia, este actúa como el órgano ejecutivo de gobierno del sistema de investigación científica y tecnológica de la UNAH. Una vez determinado los órganos de gobierno del sistema, es requerido definir cuáles son los supuestos del modelo secuencial que se aplican en este caso:

1. Los hechos en una sociedad son interpretados en forma variada por las diferentes personas y en múltiples momentos.

2. Muchos problemas pueden resultar a partir de un mismo hecho.

3. La gente goza de diferentes grados de acceso al proceso de las políticas en el gobierno.

4. Los problemas y las demandas son constantemente definidos y redefinidos en el proceso de las políticas públicas.

5. No existe un sistema político ideal independiente de las preferencias del arquitecto de dicho sistema.

En el primer supuesto: "Los hechos en una sociedad son interpretados en forma variada por las diferentes personas y en múltiples momentos", se evidencia fácilmente en la postura de la Comisión de Transición plasmado en el Plan General de la Reforma Integral de la Universidad en los resultados esperados al 2007, todos ellos son productos esperados a corto plazo, sin embargo, no se prevé la construcción de "instituciones" que a largo plazo es lo que puede y da sostenibilidad a los sistemas. ¿Servirá de mucho contar con ejes de educación en investigación en todas sus carreras 0 institutos de investigación sin profesores formados en investigación y que realicen investigación?, ¿plantear un sistema de incentivos asegura que los profesores universitarios investiguen en sus distintas disciplinas?

El primer supuesto del modelo secuencial se da por hecho en el caso de la política de investigación científica, desarrollo tecnológico e innovación de la UNAH, debido a que 
en dos momentos históricos distintos los hechos fueron interpretados de distinta manera: en un primero era necesario que se concretaran tareas que pudiesen evidenciar un trabajo a favor de la investigación científica como función básica, y en un segundo momento se interpretó la necesidad de dar sostenibilidad al sistema a través de la construcción de instituciones. Si se realiza un balance de lo planificado por la Comisión de Transición en 2006 y los resultados obtenidos en estos diez años pueden resumirse que existen resultados intermedios o capacidades intermedias que serían requeridas para llegar al resultado final.

\section{Tabla 3. Productos intermedios en la planificación en investigación por la Comi- sión de Transición UNAH 2005}

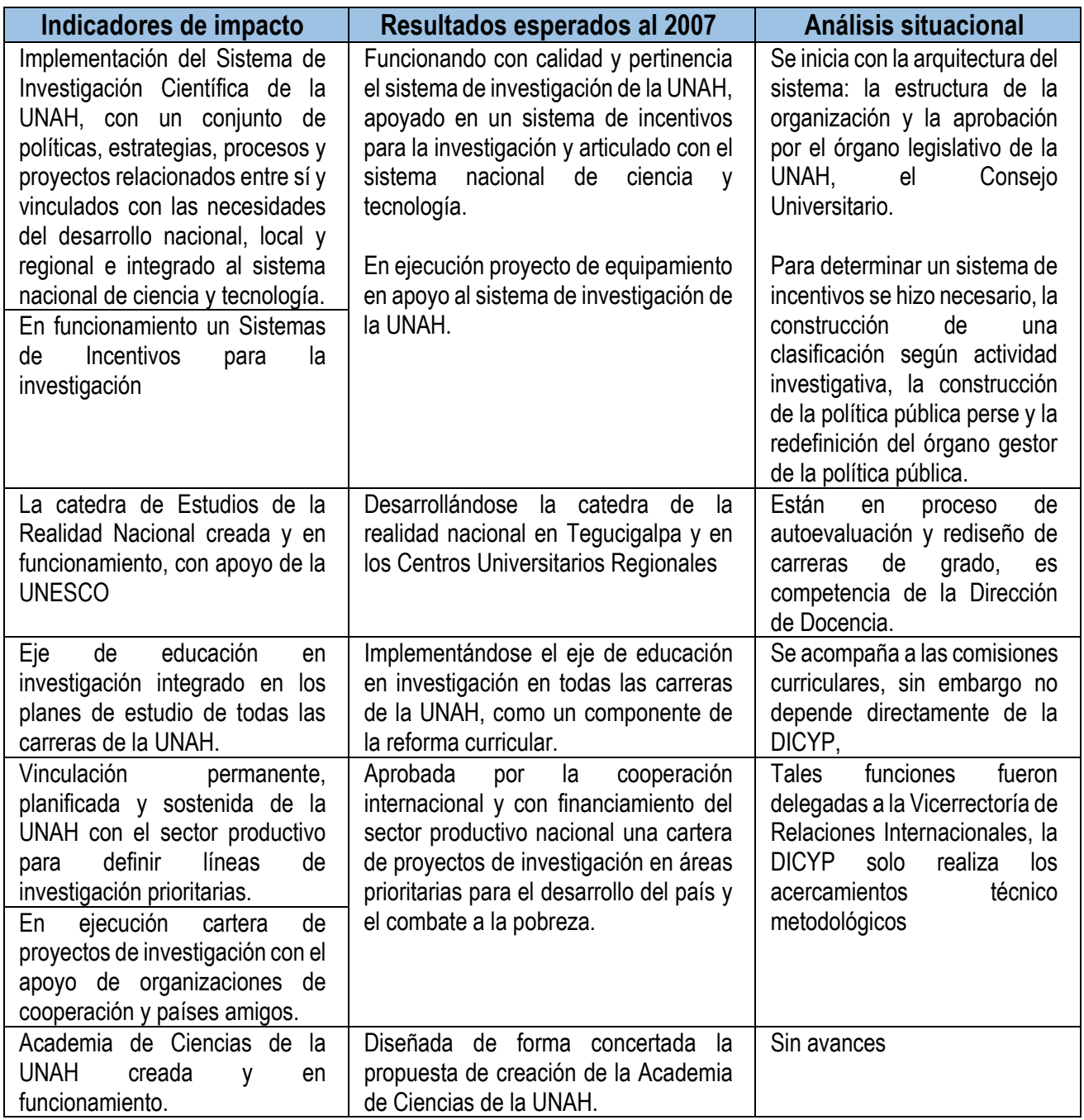




\begin{tabular}{|c|c|c|}
\hline Indicadores de impacto & Resultados esperados al 2007 & Análisis situacional \\
\hline $\begin{array}{l}\text { Instituto de Investigación } \\
\text { Educativa creado y en } \\
\text { funcionamiento }\end{array}$ & & $\begin{array}{l}\text { Los institutos de investigación } \\
\text { deberán cumplir con los } \\
\text { requerimientos estatuarios } \\
\text { aprobados por el órgano } \\
\text { legislativo, uno es contar con } \\
\text { profesores investigadores y } \\
\text { hasta el momento la facultad } \\
\text { que corresponde su dirección } \\
\text { carece de ellos. }\end{array}$ \\
\hline $\begin{array}{l}\text { Instituto de Investigaciones en } \\
\text { Ciencias de la tierra en } \\
\text { funcionamiento }\end{array}$ & $\begin{array}{l}\text { Elaborada la propuesta de creación del } \\
\text { instituto de investigaciones en Ciencias } \\
\text { de la Tierra }\end{array}$ & $\begin{array}{l}\text { Instituto creado y en } \\
\text { funcionamiento }\end{array}$ \\
\hline $\begin{array}{l}\text { Programa de re vinculación de } \\
\text { científicos hondureños en el } \\
\text { exterior diseñado y en } \\
\text { funcionamiento, con el apoyo de } \\
\text { la UNESCO }\end{array}$ & & Sin avances \\
\hline $\begin{array}{l}\text { Programa de certificación de } \\
\text { investigadores creado y en } \\
\text { funcionamiento }\end{array}$ & & $\begin{array}{l}\text { Aprobación del procedimiento } \\
\text { de clasificación de profesores } \\
\text { investigadores, en ejecución }\end{array}$ \\
\hline $\begin{array}{l}\text { Carrera de investigador } \\
\text { universitario implementada }\end{array}$ & & Sin avances \\
\hline $\begin{array}{l}\text { Participación de la UNAH en } \\
\text { proyectos de investigación } \\
\text { colaborativos y multicéntricos }\end{array}$ & & $\begin{array}{l}\text { Se vincula con organizaciones } \\
\text { estatales, productivas y } \\
\text { sociales }\end{array}$ \\
\hline $\begin{array}{l}\text { La editorial universitaria } \\
\text { reorientada y apoyada para que } \\
\text { sustente la estrategia de } \\
\text { divulgación del conocimiento } \\
\text { generado en la UNAH a nivel } \\
\text { nacional e internacional. }\end{array}$ & $\begin{array}{l}\text { En ejecución } \\
\text { proyecto de } \\
\text { fortalecimiento de } \\
\text { Universitaria }\end{array}$ & $\begin{array}{l}\text { No depende directamente de } \\
\text { la DICYP }\end{array}$ \\
\hline
\end{tabular}

Fuente: (Comisión de Transición UNAH , 2005)

El segundo supuesto que analizamos es "Muchos problemas pueden resultar a partir de un mismo hecho", sin duda el mayor problema de la UNAH tal como lo denuncia Olvin Rodríguez es la legitimidad del gobierno universitario, que incluía la concentración del poder en ciertas figuras, muchas de ellas en órganos unipersonales. Es por ello que se fortalece el gobierno universitario para darle legitimidad, desconcentrando y descentralizando funciones, dividiendo las funciones legislativas y ejecutivas en los sistemas para darle celeridad a las demandas sociales tal cual está sucediendo; sin embargo es evidente que las viejas prácticas institucionalizadas se resisten a desaparecer.

El sistema de investigación científica y tecnológica de la UNAH posee un gobierno jerárquico, esto significa por distintas capas que responden a un órgano máximo de 
conducción pero que este mismo delega en funciones ejecutivas y solo se convierte en un órgano de dirección y control, no ejecutor, con el fin de que la legitimidad de su accionar sea cercano a sus miembros.

El supuesto "La gente goza de diferentes grados de acceso al proceso de las políticas en el gobierno", define un rumbo en la construcción de una política pública, en este caso la política pública en investigación científica de la UNAH, la cual su construcción se basa en el principio de la igualdad no la equidad, ya que para el gobierno del sistema de investigación científica y tecnológica sus miembros poseen el mismo acceso a los beneficios de la política pública, diferenciando los niveles de capacidades para accesar a ellos, teniendo como premisa que todos sus miembros pueden alcanzar esa misma capacidad.

Por lo cual la política pública en investigación científica y tecnológica es una política regulatoria distributiva. Planificando y desarrollando acciones y estrategias para aquellos segmentos de miembros que requieren fortalecer sus capacidades para accesar a los beneficios de la política perse, esto como un democratizador de la política pública.

Los distintos accesos que tiene la población meta (la comunidad universitaria) a la política pública en investigación científica de la UNAH se enmarcan en acciones de política derivados de los ejes de política pública, como se afirma anteriormente la política pública en investigación científica de la UNAH se construyó basado en el principio de igualdad con el fin de democratizar el sistema.

"Los problemas y las demandas son constantemente definidos y redefinidos en el proceso de las políticas públicas". Este supuesto se lleva a todo lo largo de la política pública, ya que desde que la Comisión de Transición nombra como Directora de Investigación Científica a la profesora Leticia Salomón en 2006, quien hasta la fecha ha dirigido varios procesos de definición y redefinición de la política pública, teniendo distintos cortes en el tiempo.

Si bien una política pública va más allá de una norma, para las organizaciones anárquicas como son las universidades, las normas son requeridas para evitar contratiempos, dificultades, rezagos y otros. Es por ello que tal como lo muestra la ilustración 2 , se han consensuado, aprobado y publicado normas que construyen instituciones además de la redefinición de algunas de ellas basados en el primer supuesto del modelo secuencial. 


\section{Tabla 4: Acciones de la política pública en investigación científica de la UNAH}

\begin{tabular}{|c|c|c|}
\hline Eje de política & Acciones de política & $\begin{array}{l}\text { Segmento poblacional que } \\
\text { se pretende fortalecer }\end{array}$ \\
\hline \multirow{5}{*}{$\begin{array}{l}\text { Fomento a la } \\
\text { investigación científica }\end{array}$} & Fondos concursables para realizar investigación & Profesores y estudiantes \\
\hline & $\begin{array}{l}\text { Incentivos sociales y económicos por actividad } \\
\text { investigadora (premios) }\end{array}$ & Profesores y estudiantes \\
\hline & $\begin{array}{l}\text { Reconocimiento de tiempo efectivo para desarrollar } \\
\text { investigación }\end{array}$ & Profesores \\
\hline & $\begin{array}{l}\text { Fondos para la divulgación de resultados de } \\
\text { investigación en eventos científicos internacionales }\end{array}$ & Profesores \\
\hline & $\begin{array}{l}\text { Fondos concursables para desarrollo de gestión de } \\
\text { la investigación del gobierno del sistema }\end{array}$ & Gobierno del sistema \\
\hline \multirow[t]{3}{*}{$\begin{array}{l}\text { Publicación, difusión y } \\
\text { comunicación }\end{array}$} & $\begin{array}{l}\text { Espacios internos para la publicación de resultados } \\
\text { de investigación (revistas, boletines, periódicos) }\end{array}$ & Profesores y estudiantes \\
\hline & $\begin{array}{l}\text { Espacios internos para la divulgación de resultados } \\
\text { de investigación (congresos) }\end{array}$ & Profesores y estudiantes \\
\hline & $\begin{array}{l}\text { Reconocimiento y clasificación según resultados } \\
\text { de actividad investigativa (catálogo de profesores } \\
\text { investigadores con publicaciones efectuadas) }\end{array}$ & Profesores \\
\hline $\begin{array}{l}\text { Protección } \\
\text { resultados } \\
\text { investigación }\end{array}$ & $\begin{array}{l}\text { Registro de marcas, derechos de autor y derechos } \\
\text { de propiedad industrial }\end{array}$ & Profesores \\
\hline $\begin{array}{l}\text { Capacitación } \\
\text { investigación }\end{array}$ & $\begin{array}{l}\text { Formación permanente en metodología de } \\
\text { investigación (diplomados, cursos, inducciones y } \\
\text { socializaciones) }\end{array}$ & Profesores y estudiantes \\
\hline \multirow[t]{2}{*}{$\begin{array}{l}\text { Gestión de la } \\
\text { investigación }\end{array}$} & $\begin{array}{l}\text { Reuniones programadas de evaluación de avances } \\
\text { en la implementación de la política pública en las } \\
\text { distintas capas del gobierno del sistema }\end{array}$ & Gobierno del sistema \\
\hline & $\begin{array}{l}\text { Promoción del fortalecimiento de la estructura } \\
\text { organizacional del gobierno del sistema }\end{array}$ & $\begin{array}{l}\text { Facultades y } \text { Centros } \\
\text { Universitarios Regionales }\end{array}$ \\
\hline
\end{tabular}

El último supuesto en esta ocasión es "No existe un sistema político ideal independiente de las preferencias del arquitecto de dicho sistema", es por ello que la constancia en los cambios y ajustes que ha tenido la política pública en investigación de la UNAH son pertinentes, a pesar de que los indicadores que la Comisión de Transición planteo en el año 2006 no fueron cubiertos en su totalidad, porque en aquel momento histórico se desconocía con certeza la situación real de la investigación científica y la gestión de la investigación en la Universidad.

Posteriormente la Rectoría de la UNAH, decidió junto a la Vicerrectoría Académica, quien por mandato estatuario corresponde la dirección de los sistemas académicos, fusionar las Direcciones de Investigación Científica y del Sistema de Estudios de Posgrado, en un órgano gestor fortalecido que tuviese las facultades de potenciar ambos sistemas en un marco de cooperación y no de subordinación. 
Ilustración 2: Definición y redefinición de instituciones en el sistema de investigación científica y tecnológica

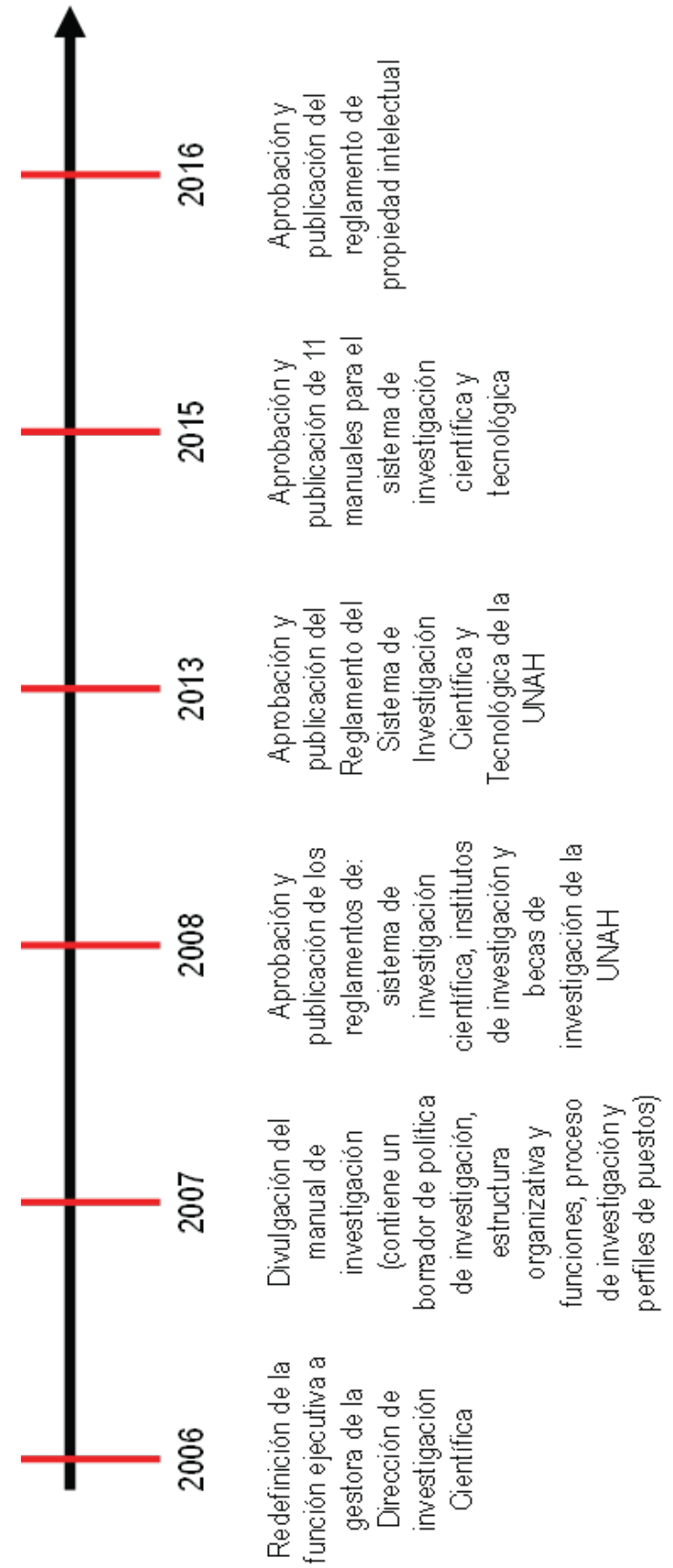

Fuente: Construcción propia con referentes de la DICYP 
Es así como se constituye un único órgano gestor de la política pública en investigación científica y de los estudios de posgrado, denominada como dirección de investigación científica y posgrado, manteniendo la independencia de los sistemas pero logrando que las intercepciones entre ellos sean pronunciadas.

\section{CONCLUSIONES}

Si bien, se contempla en el análisis que la política pública en investigación científica de la UNAH ha tenido una implementación sin shocks (solo los suscitados en el país, como el golpe de Estado en 2009 y la crisis financiera en 2008) que pudieran repercutir en cambios de política pública agresivos, coaliciones promotoras divergentes en la implementación, o problemas de financiación debido a que su fuente es procedente del erario público, eso afirma que dicha política pública goza de un compromiso de todos los miembros de la comunidad universitaria.

La legitimidad del gobierno del sistema es considerable, ya que son miembros del mismo sistema quienes lo conforman y tienen las atribuciones estatuarias para la construcción y modificación de la política pública, esto es una fortaleza, a pesar que la etapa de la formulación de la política pública y las alternativas de solución al problema publico fueron construidas en un momento histórico de mucha conflictividad y no en las mejores condiciones del contexto, debido a que el periodo de mandato de la Comisión de Transición era moderadamente corto.

Aunque no se concluyeron con las tareas encomendadas por la Comisión de Transición planteadas en el 2005 en el Plan General de la Reforma Integral de la UNAH, se desarrollaron acciones de política encaminadas a tales productos, procurando la sostenibilidad de las mismas.

Se han focalizado los esfuerzos de política en fortalecer el gobierno del sistema y "democratizar" el acceso a los beneficios de las acciones de política, más que en la expansión del sistema en sí, podría decirse que antes del desarrollo del sistema se ha considerado su fortalecimiento. Se deberá en todo sentida afianzar las acciones de política como la carrera de profesor investigador para proseguir con las acciones subyacentes a la misma.

Vincular la editorial universitaria y el sistema bibliotecario es una tarea fundamental y esta pendiente, no solo fortalecer los mismos de manera independiente sino tal, como 
se vincularon los sistemas de investigación científica y del sistema de estudios de posgrado, es necesario vincular el sistema editorial y sistema bibliotecario con los dos anteriores, su potenciación seria creciente y se aprovecharía al máximo los pocos recursos financieros con que cuenta la Universidad para la gestión académica en los cuatro sistemas en mención.

\section{REFERENCIAS BIBLIOGRÁFICAS}

http://www. elheraldo.hn/opinion/columnas/988888-469/algunas-causas-de-la-crisis-en-la-unah

Comisión de Transición UNAH . (2005). Plan General de la Reforma Integral de la Universidad . Tegucigalpa . 